\title{
3D NONLINEAR PARALLEL FEM ANALYSIS FOR SEISMIC EARTH PRESSURES OF A SATURATED SOIL LAYER
}

\author{
Makoto KAWAMURA ${ }^{1}$, Jafril TANJUNG ${ }^{2}$ \\ ${ }^{1}$ Member of JSCE, Dr. Eng., Professor, Dept. of Architecture and Civil Eng., Toyohashi University of Technology \\ (1-1 Hibarigaoka, Tempaku-cho, Toyohashi 441-8580, Japan) \\ ${ }^{2}$ Student Member of JSCE, Graduate Student, Dept. of Mech. Struc. Sys. Eng., Toyohashi University of Technology \\ (1-1 Hibarigaoka, Tempaku-cho, Toyohashi 441-8580, Japan)
}

\begin{abstract}
In this study a 3D nonlinear parallel FEM formulation was derived for dynamic soil structure interaction problems. To express the nonlinear property of the saturated soil the simplified bounding surface model was used referring the Wolf and Crouch's study. In the implementation of the parallel processing algorithm for the 3D nonlinear FEM formulation, Domain Decomposition Method and Conjugate Gradient Method were applied. To see the validity of the proposed parallel analysis a seismic earth pressure test using a shaking table was simulated. The numerical results for the seismic active earth pressures and dynamic pore water pressures acting on the wall coincide well with those of the experiment.
\end{abstract}

Key Words : parallel processing, dynamic response, earth pressure, saturated layer, bounding surface

\section{INTRODUCTION}

As the severe damage of the port of Kobe in the 1995 Hyogo-Ken Nanbu earthquake, damages of water front structures such as quay walls are often encountered in the great earthquakes. To understand the dynamic behaviors of the water front structures which retain saturated soil layers, dynamic interaction between the water front structures and saturated soil layers should be analyzed more exactly. There exist two problems for analysis of the dynamic interaction. The first problem is how to idealize the saturated soil layer. It is required to assume the soil layer as the composite of the soil grains and the pore water and to solve a fluid and solid coupling problem. It is necessary to use an appropriate material constitutive model for the soil grains, which is able to represent the nonlinear stress-strain relationship and the loading-unloading hysteresis loops. To solve the first problem, the bounding surface plasticity model was introduced referring the previous studies by Dafalias ${ }^{1)}$ and Wolf-Crouch ${ }^{2}$. In this model the change of plastic strain during cyclic loading is evaluated according to the relative position of the current stress point on the loading surface inside the bounding surface. The model is applicable for both of clays and sands by unifying the parameters in the single frame of a constitutive model. In the model developed by Wolf and Crouch the bounding surface is defined by three different equations. In this study the bounding surface is determined by a single function. The number of the parameter to specify the nonlinear relation is reduced in this study.

The second problem is how to reduce heavy loads on the computation processes. Long computation time and large computer memory are needed to solve three dimensional, coupling problem applying iterative method for nonlinear analysis and time history response analysis for a recorded earthquake motion. To overcome this problem the authors proposed the parallel computation of the FEM analysis for the dynamic response of a saturated soil layer which has a linear stress-strain relation ${ }^{3)}$. The proposed parallel FEM analysis was conducted by allocating blocks of the 
computation into several individual processors. The Domain Decomposition Method (DDM) ${ }^{4,5)}$ was used to partite the whole domain of the analytical model. As this parallel computation is more effective for the nonlinear case, the parallel FEM formulation is extended to the nonlinear problem in this study. The nonlinear problem is iteratively solved by the modified Newton-Raphson scheme ${ }^{6}$.

In the previous studies parallel FEM analysis was applied for the linear dynamic problem of solid materials like metals. In this study the nonlinear dynamic problem for saturated soils was solved first by parallel 3D FEM considering soil structure interactions and coupling of two phase material.

In this paper the authors propose parallel 3D FEM formulations to analyze more exactly the dynamic soil-structure interaction problem. The validity and efficiency of the proposed FEM analysis is investigated by comparing the analytical results with the experimental results of a shaking table test for the seismic earth pressure of a saturated soils.

\section{FORMULATION FOR NUMERICAL ANALYSIS}

\section{(1) Governing equations}

The governing equations are derived by assuming that the saturated soils are composed of the soil grains and the pore water. The density of the saturated soil, $\rho$ is expressed by the density of the soil grains $\rho_{g}$ and the fluid density $\rho_{f}$, as schematically shown in Fig. 1.

$$
\rho=(l-n) \rho_{g}+n \rho_{f}
$$

where $n$ is the porosity.

Assuming infinitesimal displacement the equations of motions for saturated porous material are given as follows. ${ }^{7,8)}$

$$
\begin{aligned}
& \sigma_{i j, i}-\rho \ddot{u}_{i}-\rho_{f} \ddot{w}_{i}=0 \\
& p_{, i}-\rho_{f}\left(\ddot{u}_{i}+\frac{\ddot{w}_{i}}{n}\right)-\frac{\rho_{f} g}{k} \dot{w}_{i}=0
\end{aligned}
$$

where $\sigma_{i j}$ are the components of total stress of the

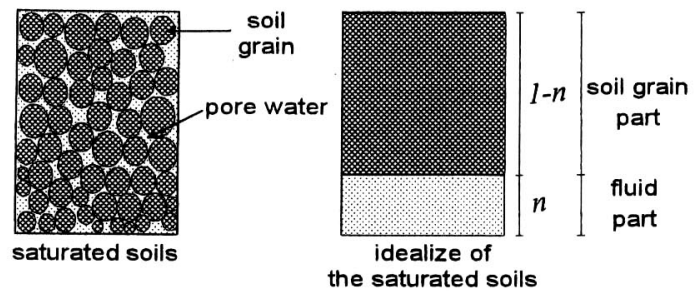

Fig. 1. Idealization of the Saturated Soils saturated soil, $p$ is the pore water pressure, $u_{i}$ and $w_{i}$ are the components of displacement of the soil grain and the relative displacement of the pore water to $u_{i}$, respectively. The superposed dot implies time derivative, ()$_{i}$ denotes the derivative with respect to coordinates $x_{i}$. For $\sigma_{i j}$ and $p$ tensile stress are defined positive in this paper. $k$ is the permeability and $g$ is the gravitational acceleration. As Darcy's law, the relation $w=k \cdot i$ is used, where $i$ is hydraulic gradient.

\section{(2) Stress strain relations}

The stress strain relation for the total stress increment $\dot{\sigma}_{i j}$ and the pore water increment $\dot{p}$ are given as follows.

$$
\begin{aligned}
& \dot{\sigma}_{i j}=D_{i j k l} \dot{\varepsilon}_{k l}+\alpha^{2} \delta_{i j} K_{g f} \delta_{k l} \dot{\varepsilon}_{k l}+\alpha K_{g f} \delta_{i j} \dot{\zeta} \\
& \dot{p}=K_{g f}\left(\alpha \dot{\varepsilon}_{i i}+\dot{\zeta}\right)
\end{aligned}
$$

where $D_{i j k l}$ represents the stress strain relation of soil skeleton, $\alpha$ and $K_{g f}$ are the values related to the bulk modulus of the soil grain and the pore water. In the above equations the effect of the pore water pressure on deformation of the soil skeleton is considered.

The strain $\varepsilon_{i j}$ and $\zeta$ are defined in the following equations.

$$
\begin{aligned}
& \varepsilon_{i j}=\frac{1}{2}\left(u_{i, j}+u_{j, i}\right) \\
& \zeta=w_{i, i}
\end{aligned}
$$

$\varepsilon_{i j}$ represents the strain of the soil skeleton and $\zeta$ is the volumetric strain of pore water.

Elastoplastic stress strain relation $D_{i j k l}^{e p}$ is expressed in the following equation. ${ }^{2)}$

$$
D_{i j k l}^{e p}=D_{i j k l}^{e}-u(L) \frac{D_{i j m n}^{e} P_{m n} Q_{p q} D_{p q k l}^{e}}{H+Q_{a b} D_{a b c d}^{e} P_{c d}}
$$

where

$$
\begin{aligned}
& L=\frac{Q_{i j} D_{i j k l}^{e} \dot{\varepsilon}_{k l}}{H+Q_{a b} D_{a b c d}^{e} P_{c d}} \\
& P_{i j}=\frac{\partial g_{p}}{\partial \sigma_{i j}^{\prime}} \quad ; \quad Q_{i j}=\frac{\partial f}{\partial \sigma_{i j}^{\prime}}
\end{aligned}
$$

where $D_{i j k l}^{e}$ represents the elastic stress strain relation, $H$ is the plastic hardening modulus, $g_{p}$ and $f$ are the plastic potential and the yield surface. $u(L)$ is the heavy-step function, $u(L)=1$ for $L \geq 0$ and $u(L)=0$ for $L<0$.

The above relations are derived for the general type of yield functions. In the bounding surface model, these relations are applied not only for the bounding surface but also for the loading surface inside the bounding surface. 
To specify the relations in Eq. (5) in detail, the elastic stress-strain relation $D_{i j k l}^{e}$, the failure surface $F_{f}$, the bounding surface $F_{b}$, the loading surface $f_{L}$ and the plastic strain potential $g_{p}$ should be determined. The form of the functions mentioned above is explained in the followings.

\section{Elastic stress-strain relation} as

The elastic stress-strain relation $D_{i j k l}^{e}$ is expressed

$$
D_{i j k l}^{e}=2 G \delta_{i k} \delta_{j l}+\left(K-\frac{2}{3} G\right) \delta_{i j} \delta_{k l}
$$

in which $K$ is bulk modulus and $G$ is shear modulus.

The bulk modulus $K$ is expresses as

$$
\frac{K}{p_{a}}=\frac{1+e_{i n}}{\kappa}\left(\frac{I}{3 p_{a}}\right)^{0.5}
$$

in which $e_{i n}$ is the initial void ratio, $\kappa$ is the slope of the swelling process in the consolidation curve, $p_{a}$ is the atmosphere pressure, $I$ is the first invariant of stress $\left(I=\sigma_{i j}^{\prime}\right)$.

Assuming a constant Poisson's ratio $v$, the shear modulus $G$ is written in the following expression.

$$
G=\frac{3(1-2 v)}{2(1+v)} K
$$

\section{Failure surface}

The failure surface is represented by an elliptic cone in the stress space as proposed by Wolf and Crouch $^{2)}$. The shape of the cone is controlled by the Lode angle $\theta$ and the friction angle $\phi_{c r}$. The critical state line in the meridional section is then defined as follows.

$$
\varsigma_{c r}=\rho_{c} \frac{2 \operatorname{Sin} \phi_{c r}}{\sqrt{3}\left(3-\operatorname{Sin} \phi_{c r}\right)}
$$

where $\rho_{c}$ is the elliptic function and function of $\theta$. The detail form of $\rho_{c}$ is written in Wolf and Crouch's paper $^{2)}$

The failure surface $F_{f}$ is defined as follows.

$$
F_{f}=-\varsigma_{c r} I+J=0
$$

where $J$ is the second invariant of deviatoric stress.

$$
J=\sqrt{\frac{1}{2} s_{i j} s_{i j}}=\sqrt{J_{2}}
$$

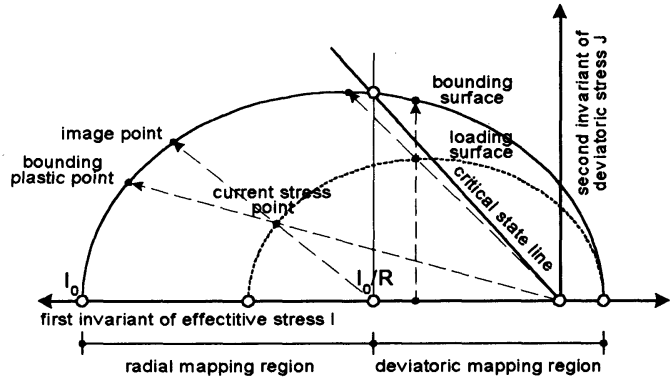

Fig.2 Single elliptic bounding surface model

The single elliptic surface with parameters $R, \varsigma_{c r}$ and $I_{0}$ are used as the bounding surface in this study. The parameter $R$ controls the aspect ratio of the ellipse, as shown in Fig. 2. The form of the bounding surface $F_{b}$ is defined as the following equation.

$$
F_{b}=\bar{J}^{2}-\frac{\left(\varsigma_{c r} I_{0}\right)^{2}}{R^{2}}\left(1-\frac{1}{(R-1)^{2}}\left(\frac{R \bar{I}}{I_{0}}-1\right)^{2}\right)=0
$$

$I_{0}$ is the maximum isotropic compression stress in the previous stress history. The size of the bounding surface is defined by $I_{0}$.

The evolution of the size of the bounding surface is written as follow.

$$
\frac{\dot{I}_{0}}{p_{a}}=\frac{1+e_{i n}}{\lambda-\kappa}\left(\frac{I_{0}}{3 p_{a}}\right) \dot{\varepsilon}_{v}^{p}
$$

where $\lambda$ is the compression index of the normally consolidation curve. $\dot{\varepsilon}_{v}^{p}$ is the plastic volumetricstrain rate.

\section{Loading surface}

The shape of the loading surface is similar with the bounding surface form. The plastic condition of the loading surface, which is located inside the bounding surface, should be related to the plastic condition on the bounding surface. The relation is given by mapping the current stress point on the image point of the bounding surface, as shown in Fig. 2. In order to capture the wide range of the soil responses due to loading histories, the combination of the radial and deviatoric mapping rules have been used in this study.

The radial and deviatoric mapping rules are written as follows.

$$
\begin{array}{ll}
\bar{I}=\left(\frac{I_{0}}{R}\right)+\beta\left(I-\frac{I_{0}}{R}\right) & \bar{J}=\beta J \\
\bar{I}=I & \bar{J}=\beta J
\end{array}
$$

in which $\beta$ is the similarity ratio. 
The equations which define the loading surfaces in the radial and deviatoric mapping regions can be obtained by substituting Eqs. (15) and (16) into the bounding surface equation, Eq. (13), respectively.

$$
\begin{aligned}
& f_{L}=\beta^{2} J^{2}-\frac{\left(\varsigma_{c r} I_{0}\right)^{2}}{R^{2}}\left(1-\frac{\beta^{2}}{(R-1)^{2}}\left(\frac{R I}{I_{O}}-1\right)^{2}\right)=0 \\
& f_{L}=\beta^{2} J^{2}-\frac{\left(\varsigma_{c r} I_{0}\right)^{2}}{R^{2}}\left(1-\frac{1}{(R-1)^{2}}\left(\frac{R I}{I_{0}}-1\right)^{2}\right)=0
\end{aligned}
$$

The similarity ratio is obtained by solving the Eqs. (17) and (18) for a given stress state.

\section{Potential surface}

The potential surface $g_{p}$ is assumed as the similar form with the bounding surface.

$$
g_{p}=J^{2}-\frac{\left(\varsigma_{\text {crg }} I_{0 g}\right)^{2}}{R_{g}{ }^{2}}\left(1-\frac{1}{\left(R_{g}-1\right)^{2}}\left(\frac{R_{g} I}{I_{0 g}}-1\right)^{2}\right)=0
$$

in which subscript $g$ denotes the parameters related to the potential surface $g_{p}$. All parameters except $I_{0 g}$ are same with those of the bounding surface. $I_{O_{g}}$ is scaled such that the current stress point lies on the potential surface.

To determine the unit vector of the plastic strain rate $\boldsymbol{P}$ easily, the deviatoric component of the unit vector of the loading direction $Q_{s}$ is assumed to coincide with the deviatoric component of the unit vector Ps.

Although the bounding surface, the loading surface and the potential surface are the similar elliptic form, these surfaces are not geometrically identical and the responses of the soil are obtained based on the non-associated flow rule.

\section{Plastic hardening modulus}

The plastic hardening modulus $H$ on the loading surface is defined referring the value of the plastic hardening modulus $\bar{H}$ on the bounding surface.

$$
H=\bar{H}+H_{f}
$$

Assuming that the hardening function is $I_{0}, \bar{H}$ can be written as follows.

$$
\bar{H}=-\frac{1+e_{i n}}{\lambda-\kappa} I_{0} \frac{\partial F}{\partial I_{0}} P_{i i}
$$

$\partial F / \partial I_{0}$ is evaluated for the bounding plastic point on the bounding surface. The bounding plastic point is determined by projecting the current stress point from the origin of the stress space onto the bounding surface as shown in Fig. 2.

To determine the bounding plastic point, the following mapping rule is used.

$$
\begin{gathered}
\bar{I}=\beta I \quad \bar{J}=\beta J \\
f_{L}=\beta^{2} J^{2}-\frac{\left(\varsigma_{c r} I_{0}\right)^{2}}{R^{2}}\left(1-\frac{1}{(R-1)^{2}}\left(\frac{\beta R I}{I_{0}}-1\right)^{2}\right)=0
\end{gathered}
$$

$H_{f}$ is calculated using the following equations, developed by Wolf and Crouch ${ }^{2)}$.

$$
\begin{aligned}
& H_{f}=\frac{1+e_{i n}}{\lambda-\kappa} \frac{h}{\left\langle\beta /(\beta-1)-s_{e}\right\rangle}\left(\frac{I_{0}}{3 p_{a}}\right)^{1.5} \\
& h=r h_{c}\left(\frac{J}{J_{I}}\right)^{0.02}+\left(1-\left(\frac{J}{J_{l}}\right)^{0.02}\right) \frac{h_{c}+h_{e}}{2}(24 b) \\
& J_{1}=\frac{S_{c r} I_{0}}{R} \\
& r=\frac{2\left(h_{e} / h_{c}\right)}{1+\left(h_{e} / h_{c}\right)-\left(1-\left(h_{e} / h_{c}\right)\right) \operatorname{Sin} 3 \theta} \\
& h_{c}=h_{c l}-\left\langle 1-\frac{I R}{I_{0}}\right\rangle\left(h_{c 1}-h_{c 2}\right) \\
& h_{e}=h_{e l}-\left\langle 1-\frac{I R}{I_{0}}\right\rangle\left(h_{e l}-h_{e 2}\right)
\end{aligned}
$$

\section{(3) Nonlinear dynamic parallel FEM analysis}

\section{a) Domain Decomposition}

In the Domain Decomposition Method (DDM) the analytical model is separated into several subdomains and the FEM analysis of the subdomains are performed parallel solving the interface problems among the subdomains. As an example, a domain $\Omega$ is decomposed into two subdomains $\Omega^{(1)}$ and $\Omega^{(2)}$ as shown in Fig. 3. $\Gamma^{(2)}$ is the interface between $\Omega^{(1)}$ and $\Omega^{(2)}$. In each subdomain the fundamental relations, Eqs. (2a) and (2b), and the constitutive relations, Eqs. (3a) and (3b) are satisfied. However, to enforce their continuity on their interface, the additional boundary conditions for the interface are required.

$$
\left\{\begin{array}{ll}
\lambda_{i j}^{u} & n_{j} \\
\lambda_{i j}^{w} & n_{j}
\end{array}\right\}^{(1)}+\left\{\begin{array}{ll}
\lambda_{i j}^{u} & n_{j} \\
\lambda_{i j}^{w} & n_{j}
\end{array}\right\}^{(2)}=0 \text { on } \Gamma^{(12)}
$$

and

$$
\left\{\begin{array}{l}
u_{i} n_{j} \\
w_{i} n_{j}
\end{array}\right\}^{(1)}=\left\{\begin{array}{l}
u_{i} n_{j} \\
w_{i} n_{j}
\end{array}\right\}^{(2)} \text { on } \Gamma^{(12)}
$$

where $\lambda_{i j}$ is a traction tensor and $n_{j}$ is an outer normal vector. The superscripts $u$ and $w$ denote the soil 


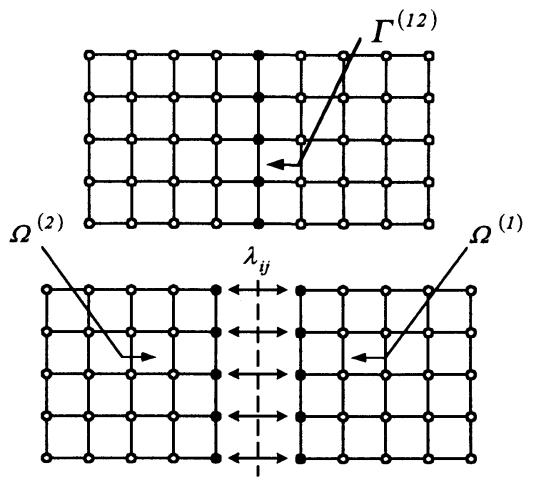

Fig.3 Decomposition into two subdomains

skeleton and the fluid part respectively.

To obtain the solution for the whole domain, the displacement based weighted residual formulation for Eqs. (2a), (2b) and (25), are led as follows.

$$
\begin{aligned}
& \sum_{s=1}^{2}\left[\int_{\Omega^{(s)}}\left\{\begin{array}{ll}
\delta u_{i} & \delta w_{i}
\end{array}\right\}^{(s)}\left\{\begin{array}{c}
\sigma_{i j, j} \\
p_{, i}
\end{array}\right\}^{(s)} d \Omega^{(s)}\right. \\
& -\int_{\Omega^{(s)}}\left\{\delta u_{i} \quad \delta w_{i}\right\}^{(s)}\left\{\left[\begin{array}{cc}
\rho & \rho_{f} \\
\rho_{f} & \frac{1}{f} \rho_{f}
\end{array}\right]\left\{\begin{array}{l}
\ddot{u}_{i} \\
\ddot{w}_{i}
\end{array}\right\}\right\}^{(s)} d \Omega^{(s)} \\
& \left.-\int_{\Omega^{(s)}}\left\{\delta u_{i} \quad \delta w_{i}\right\}^{(s)}\left\{\left[\begin{array}{cc}
0 & 0 \\
0 & \frac{\rho_{f} g}{k}
\end{array}\right]\left\{\begin{array}{l}
\dot{u}_{i} \\
\dot{w}_{i}
\end{array}\right\}\right\}^{(s)} d \Omega^{(s)}\right] \\
& +\int_{\Gamma^{(12)}}\left\{\delta u_{i} \delta w_{i}\right\}^{(12)}\left\{\left\{\begin{array}{ll}
\lambda_{i j}^{u} & n_{j} \\
\lambda_{i j}^{w} & n_{j}
\end{array}\right\}^{(1)}+\right. \\
& \left.\left\{\begin{array}{ll}
\lambda_{i j}^{u} & n_{j} \\
\lambda_{i j}^{w} & n_{j}
\end{array}\right\}\right\} d \Gamma^{(12)}=0
\end{aligned}
$$

where $s$ denotes the number of subdomains. For the inter-subdomains constraint the following equations are given.

$$
\begin{array}{r}
\int_{\Gamma^{(12)}}\left\{\delta \lambda_{i j}^{u} n_{j} \delta \lambda_{i j}^{w} n_{j}\right\}^{(12)}\left\{\left\{\begin{array}{l}
u_{i} n_{j} \\
w_{i} n_{j}
\end{array}\right\}^{(1)}-\right. \\
\left.\left\{\begin{array}{l}
u_{i} n_{j} \\
w_{i} n_{j}
\end{array}\right\}^{(2)}\right\} d \Gamma^{(12)}=0
\end{array}
$$

\section{b) FEM formulation}

Applying the integration by part of Green's theorem to the first term of left hand side in Eq. (27) and using the interpolation function as the weight in the standard Galerkin method ${ }^{9)}$, Eqs. (27) and (28) above are converted as follows.

$$
\begin{aligned}
& \sum_{s=1}^{2}\left[\boldsymbol{M}^{(s)} \ddot{\boldsymbol{d}}^{(s)}+\boldsymbol{D}^{(s)} \dot{\boldsymbol{d}}^{(s)}+\boldsymbol{K}^{(s)} \dot{\boldsymbol{d}}^{(s)}\right]=\sum_{s=1}^{2}\left[F^{(s)}-B^{(s)^{T}} \lambda\right] \\
& \sum_{s=1}^{2} \boldsymbol{B}^{(s)} \boldsymbol{d}^{(s)}=0
\end{aligned}
$$

in which

$$
\begin{gathered}
\boldsymbol{d}^{(s)}=\left\{\begin{array}{l}
\boldsymbol{d}_{u}^{(s)} \\
\boldsymbol{d}_{w}^{(s)}
\end{array}\right\} \quad \boldsymbol{M}^{(s)}=\left[\begin{array}{cc}
M_{u}^{(s)} & \boldsymbol{M}_{c}^{(s)} \\
\boldsymbol{M}_{c}^{(s)^{T}} & \boldsymbol{M}_{w}^{(s)}
\end{array}\right] \\
\boldsymbol{D}^{(s)}=\left[\begin{array}{cc}
\boldsymbol{D}_{u}^{(s)} & 0 \\
0 & \boldsymbol{D}_{w}^{(s)}
\end{array}\right] \quad \boldsymbol{K}^{(s)}=\left[\begin{array}{cc}
\boldsymbol{K}_{u}^{(s)} & \boldsymbol{K}_{c}^{(s)} \\
\boldsymbol{K}_{c}^{(s)^{T}} & \boldsymbol{K}_{w}^{(s)}
\end{array}\right]
\end{gathered}
$$

where $M^{(s)}, D^{(s)}$ and $K^{(s)}$ are the mass, damping and stiffness matrices for each subdomains. The subscript $u$ and $w$ denotes the soil skeleton and the fluid part respectively, the subscript $c$ designates the coupling condition. The vectors $d_{u}^{(s)}$ and $d_{w}^{(s)}$ are the relative displacement of the soil skeleton and the fluid for the base movement, respectively. The vector $\lambda$ is composed of $\lambda_{i j}$ which is traction forces in the interface nodes. The matrix $B^{(s)}$ is signed Boolean matrix, which localizes a subdomain quantity to the subdomain interface. If the interior degrees of freedom are numbered first and the interface ones are numbered last, the Boolean matrix $B^{(s)}$ is expressed in the following form.

$$
\boldsymbol{B}^{(s)}=\left[\begin{array}{ll}
0 & I
\end{array}\right]
$$

where $O$ is a null matrix and $I$ is the identity matrix.

\section{c) Numerical solution}

To solve Eqs. (29a) and (29b) in time domain, the Hilber- $\alpha^{10)}$ method is used as follows.

$$
\begin{aligned}
& \ddot{\boldsymbol{d}}_{n}^{(s)}=\frac{1}{\bar{\beta} \Delta t^{2}} \Delta \boldsymbol{d}_{n}^{(s)}-\frac{1}{\bar{\beta} \Delta t} \dot{\mathrm{d}}_{n-1}^{(s)}+\left(1-\frac{1}{2 \bar{\beta}}\right) \ddot{\boldsymbol{d}}_{n-1}^{(s)} \\
& \dot{\boldsymbol{d}}_{n}^{(s)}=\frac{\bar{\gamma}}{\bar{\beta} \Delta t} \Delta \boldsymbol{d}_{n}^{(s)}+\left(1-\frac{\bar{\gamma}}{\bar{\beta}}\right) \dot{\boldsymbol{d}}_{n-1}^{(s)}+\left(1-\frac{\bar{\gamma}}{2 \bar{\beta}}\right) \Delta t \ddot{\boldsymbol{d}}_{n-1}^{(s)}(32 \\
& \boldsymbol{d}_{n}^{(s)}=\boldsymbol{d}_{n-1}^{(s)}+\Delta \boldsymbol{d}_{n}^{(s)} \\
& \quad \frac{1}{3} \leq \bar{\alpha} \leq 0 ; \bar{\gamma}=\frac{1-2 \bar{\alpha}}{2} ; \bar{\beta}=\frac{(1-\bar{\alpha})^{2}}{4}
\end{aligned}
$$

Substituting Eqs. (32c) and (32d) into Eq. (29a) and extending the number of subdomain to $N_{s}$, the fundamental equations are expressed as follows.

$$
\sum_{s=1}^{N_{s}}\left[\hat{K}_{T_{n-1}}^{(s)} \Delta d_{n}^{(s)}\right]=\sum_{s=1}^{N_{s}}\left[\Delta F_{n}^{(s)}-B^{(s)^{T}} \Delta \lambda_{n}\right]
$$


or

$$
\begin{aligned}
& \sum_{s=1}^{N_{s}}\left[B^{(s)} \hat{\boldsymbol{K}}_{T_{n-1}}^{(s)^{-1}} B^{(s)^{T}}\right] \Delta \lambda_{n}=\sum_{s=1}^{N_{s}}\left[B^{(s)} \hat{\boldsymbol{K}}_{T_{n-1}}^{(s)^{-1}} \Delta \boldsymbol{F}_{n}^{(s)}\right] \\
& \sum_{s=1}^{N_{s}} B^{(s)} \Delta d_{n}^{(s)}=0 \\
& \Delta d_{n}^{(s)}=\hat{K}_{T_{n-1}}^{(s)^{-1}}\left(\Delta F_{n}^{(s)}-B^{(s)^{T}} \Delta \lambda_{n}\right) \\
& \hat{\boldsymbol{K}}_{T_{n-1}}^{(s)}=\frac{1}{\bar{\beta} \Delta t^{2}} \boldsymbol{M}^{(s)}+\frac{\bar{\gamma}}{\bar{\beta} \Delta t} D^{(s)}+\boldsymbol{K}_{T_{n-1}}^{(s)} \\
& \Delta \hat{F}_{n}^{(s)}=\left(F_{n}^{(s)}-F_{n-1}^{(s)}\right)+\left(\frac{1}{\bar{\beta} \Delta t} M^{(s)}+\frac{\bar{\gamma}}{\bar{\beta}} D^{(s)}\right) \dot{d}_{n-1}^{(s)} \\
& +\left(\frac{1}{2 \bar{\beta}} \boldsymbol{M}^{(s)}+\left(\frac{\bar{\gamma}}{2 \bar{\beta}}-1\right) \Delta t D^{(s)}\right) \ddot{d}_{n-1}^{(s)}
\end{aligned}
$$

The vector $\Delta \lambda$ in Eq. (33b) is solved using Conjugate Gradient (CG) algorithms ${ }^{3)} . \Delta d_{n}$ is evaluated from Eq. (33d). In this study, the nonlinear stress strain relation for the soil skeleton is assumed. The solution of Eqs. (33b) and (33c) in time domain is carried out applying the modified Newton Raphson Method. In the nonlinear analysis for $k^{\text {th }}$ iteration, the incremental nodal force vector $\Delta \hat{\boldsymbol{F}}_{n}^{(s)}$ in Eqs. (33b) and (33d) is replaced by residual ${ }^{\left(k^{n}\right)} \Delta R_{n}^{(s)}$ in Eq. (34).

$$
\text { (k) } \begin{aligned}
\Delta \boldsymbol{R}_{n}^{(s)} & =\Delta \hat{\boldsymbol{F}}_{n}^{(s)}-{ }^{(k-1)} \Delta \boldsymbol{R}_{n}^{(s)} \\
& =\Delta \hat{\boldsymbol{F}}_{n}^{(s)}-\sum_{j=1}^{k-1}\left(\hat{\boldsymbol{K}}_{T_{n}}^{(s)}{ }^{(j)} \Delta \boldsymbol{d}_{n}^{(s)}\right)
\end{aligned}
$$

The iterative process is terminated when the following convergence criterion in each subdomain is satisfied.

$$
\frac{{ }^{(k)} \Delta \boldsymbol{R}_{n}^{(s)^{T}}(k) \Delta \boldsymbol{d}_{n}^{(s)}}{\Delta \hat{\boldsymbol{F}}_{n}^{(s)^{T}}(k) \Delta \hat{\boldsymbol{d}}_{n}^{(s)}} \leq \varepsilon_{c}
$$

where

$$
{ }^{(k)} \Delta \hat{\boldsymbol{d}}_{n}^{(s)}=\sum_{j=1}^{k}{ }^{(j)} \Delta \boldsymbol{d}_{n}^{(s)}
$$

The increments $\Delta \sigma_{n}^{k}$ and $\Delta p_{n}^{k}$ at the $k^{\text {th }}$ iteration in the $n^{\text {th }}$ time step are given in Eqs. (36a) and (36b).

$$
\begin{gathered}
\left\{\begin{array}{c}
\Delta \sigma_{n}^{k} \\
\Delta p_{n}^{k}
\end{array}\right\}=\left[\begin{array}{cc}
\bar{D}_{n}^{e p^{k}}+\delta \alpha^{2} K_{g f} \delta^{T} & \alpha K_{g f} \delta \\
\alpha K_{g f} \delta^{T} & K_{g f}
\end{array}\right]\left\{\begin{array}{c}
\Delta \varepsilon_{n}^{k} \\
\Delta \zeta_{n}^{k}
\end{array}\right\} \\
\bar{D}_{n}^{e p^{k}}=\frac{1}{2}\left(D_{n-1}^{e p}+D_{n}^{e p^{k-1}}\right)
\end{gathered}
$$

\section{d) Parallel computation}

In order to achieve efficient parallel computations the Domain Decomposition Method described above is implemented using Single Program Multiple Data (SPMD) programming model. The interprocessor communication is performed in the message-passing paradigm and the PVM programming libraries ${ }^{11)}$ is used as a parallel interpreter.

To form and assemble the mass matrix, damping matrix, stiffness matrix for each subdomain, the computations are executed parallel in the assignment processors. For each time step, the dynamic loading vector for each subdomain can be calculated in the parallel manner. Although the CG algorithm requires the interprocessor communication, the algorithm is more amenable to parallel manner than direct solution of Eq. (33b). For the efficient interprocessor communication a model for three-dimensional hypercube networking is considered in this paper. The explanation of the hypercube networking is described in the authors' paper ${ }^{3)}$.

The parallel computation of this study has been done on the share memory type of multiprocessor SGI Origin/2000 machine. The FORTRAN77 computer code has been applied with message passing between processors for exchanging the data.

\section{NUMERICAL ANALYSIS}

\section{(1) A model for analysis}

To evaluate the validity and the efficiency of the proposed numerical analysis, the shaking table test ${ }^{12)}$ which was performed to investigate the seismic earth pressure of the saturated sand layer, was simulated by the proposed method. In the experiment Toyoura sand was used as the backfill soil. The soil was contained every $10 \mathrm{~cm}$ deep in the steel box partially filled with the water and compacted to a dense condition. The dimensions of the steel box are $1.00 \mathrm{~m}$ high, $2.00 \mathrm{~m}$ long and $1.00 \mathrm{~m}$ wide. The height of the saturated backfill sand was $56.0 \mathrm{~cm}$. The box was subjected to a pure sinusoidal excitation with the frequency of $3 \mathrm{~Hz}$. One of the side wall of the box is equipped to move outward during the excitation and the pressures acting on the wall can be measured using the load cells as shown in Fig. 4. The velocity of the wall movement in the horizontal direction is $0.02 \mathrm{~mm} / \mathrm{sec}$. The movement of the wall was measured at the middle of the wall height. Pore water pressures are measured using pore water pressure cells attached to the surface of the moveable wall.

The model for the numerical analysis is the same size of the backfill and the one side wall can move outward. The FEM mesh of the model is shown in Fig. 5. The model was divided into eight subdomains assigned to eight processors individually without overlapping and the model was solved in parallel by the 
eight processors. To maintain the continuity conditions between the subdomains, they were linked by the interfaces expressed as the bold dashed lines. As shown in Fig.5 the whole domain is separated of eight subdomains of soil, and two walls and two joint interfaces .Each subdomain is composed of 150 hexahedra element with eight nodes and 27 integration points. The size of the subdomain was determined by trial and error to obtain stable results for the stresses.

As the numerical integration method in time domain, Hilber- $\alpha$ method was applied with the time step $\Delta t=0.01$ second. The parameters of Hilber- $\alpha$ method $\bar{\alpha}, \bar{\beta}$ and $\bar{\gamma}$ are $-0.25,0.25$ and 0.50 , respectively. The damping ratio of granular soil skeleton was assumed as 0.05 . The final solution in each step was confirmed when the modified Newton Raphson convergence criterion $\varepsilon_{c}=10^{-4}$ was satisfied.

The joint element was introduced between the backfill soil and the moveable side wall. The joint element model developed by $\mathrm{Beer}^{13)}$ for two and three-dimensional FEM analysis, was used in this analysis. The criterion for the stress and the relative displacement was defined referring the paper by Toki et al. ${ }^{14)}$. Except for the upper surface of the model and the moveable wall, both of the soil skeleton and the pore water are not allowed to move outward at the boundaries of the model. The pore water pressures on

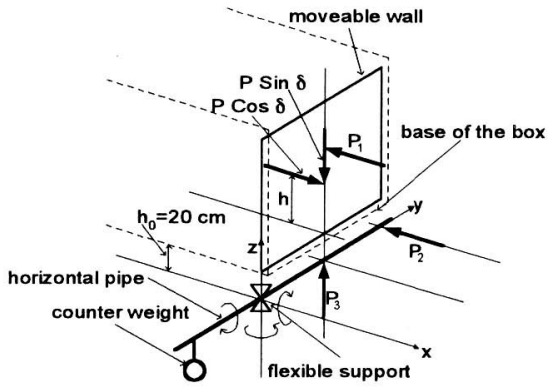

Fig. 4 Setup of the experimental model

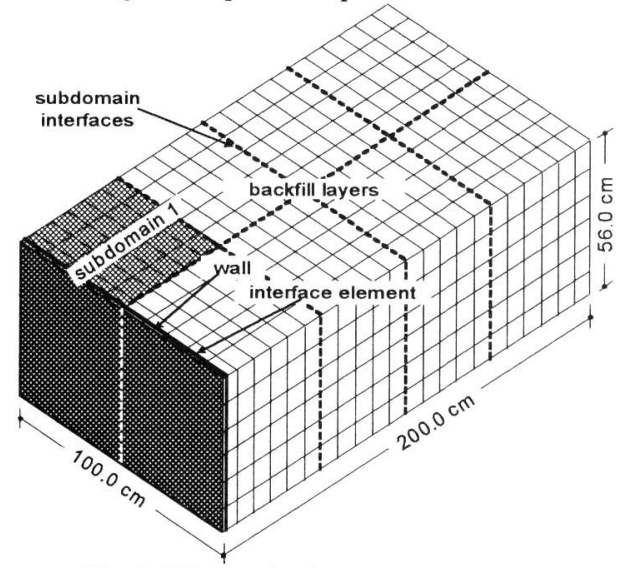

Fig. 5 FEM mesh of the numerical model the upper surface of the model are kept equal to zero during the analysis.

The horizontal acceleration is applied along the longitudinal direction and perpendicular to the wall. The stresses acting on the wall are compared with the measured loads acting on the movable wall. As the boundary condition the displacement to the outward is only fixed. The slippage between the wall and the backfill soil is allowed but the opening is not permitted at the joint interface. The initial condition for the pore water pressure is hydrostatic. The coefficient of the earth pressure at rest which is related to the initial condition for the effective stress, was specified as 0.6 based on the experimental data and the fact that in the experiment the backfill was compacted artificially as resulting in a moderate dense condition.

\section{(2) Material parameters}

To apply the bounding surface model successfully several parameters should be defined properly based on the experimental results. An experimental work for sands including Toyoura sands was conducted by Been et al. ${ }^{15)}$ to define the properties in the framework of critical state soil mechanics. Pradhan et al. ${ }^{16)}$ investigated the properties of Toyoura sand under cyclic loads through the series of laboratory tests. Kawamura ${ }^{12)}$ also tested to obtain the physical properties of saturated Toyoura sand which was used for the shaking table tests. The material properties of Toyoura sand which is the backfill sand for the numerical analysis, are defined using the experimental data by Been et al., Pradhan et al. and Kawamura. The values of the parameters are listed in Table 1. The angle of friction in the critical state $\phi_{c r}$ is used to define the failure surface. The value of $\phi_{c r}$ is approximated $31^{\circ}$ for Toyoura sand ${ }^{17)}$ and the same value was assumed for both of compression and extension meridian. The parameters $\lambda$ and $\kappa$ are the slope of the normally consolidation line and the swelling line, respectively. These values were determined by standard isotropic

\begin{tabular}{llll}
\multicolumn{4}{c}{ Table 1 Material properties of the backfill soils } \\
\hline Property & Symbol & Value & Unit \\
\hline Effective frictional angle & $\phi_{c r}$ & 31 & degree \\
Slope of isotropic cons. Line & $\lambda$ & 0.02 & \\
Slope of elastic rebound line & $\kappa$ & 0.002 & \\
Parameter def. shape of ellipse & $R$ & 2.25 & \\
Elastic nucleus parameter & $S_{e}$ & 1.0 & \\
Hardening shape factor & $h_{c l} / h_{c 2}$ & $0.02 / 0.001$ & \\
& $h_{e l} / h_{e 2}$ & $0.02 / 0.002$ & \\
Initial void ratio & $e_{i n}$ & 0.64 & \\
Poisson's Ratio & $v$ & 0.33 & \\
Bulk modulus of granular soil & $K_{\mathrm{g}}$ & $3.70 \times 10$ & $\mathrm{GPa}$ \\
Bulk modulus of pore water & $K_{f}$ & 2.08 & $\mathrm{GPa}$ \\
Density of saturated soi 1 & $\rho$ & 2.0 & $\mathrm{~g} / \mathrm{cm}^{3}$ \\
Density of pore water & $\rho_{f}$ & 1.0 & $\mathrm{~g} / \mathrm{cm}^{3}$ \\
Coefficient of permeability & $k$ & $1.2 \times 10^{-4}$ & $\mathrm{~m} / \mathrm{s}$ \\
Coefficient of Contact area & $\alpha$ & 1.0 & \\
\hline
\end{tabular}


consolidation tests. The aspect ratio of the ellipse for the bounding surface $R$ lies in the range of 1.5 to 3.0. In this analysis $R$ is specified 2.25 as the typical value for sands ${ }^{18)}$. A parameter $s_{e}$ defines the elastic nucleus where only elastic strains are developed. Plastic strains are found in a very low stress level in the case of sands. In this study $s_{e}$ is assumed to be one, so that the elastic nucleus shrinks to a point. The hardening shape factor $h_{c l / c 2}$ and $h_{e l / e 2}$ control the hardening behavior through the additive plastic modulus within the bounding surface. The hardening shape factors were determined by standard triaxial tests ${ }^{18)}$. A coefficient $\alpha$ is close to unity for most of soil-like material $^{8)} . \alpha$ was taken to unity in this analysis.

The material properties for the joint element and the steel wall are listed on Table 2 and Table 3, respectively. The values of the normal and the shear stiffness for the joint element were referred to the test performed by Hazarika and Matsuzawa ${ }^{19)}$.

\section{(3) Numerical results and discussion}

\section{a) Validation of parallel computation}

The velocity of the wall movement is $0.02 \mathrm{~mm} / \mathrm{sec}$ at the middle of the height $s_{m}$ in Fig. 6, which is the same condition with the experiment

The numerical values at the center of the elements behind the wall as shown in Fig. 7, are discussed as the typical results of the analysis. As an example,

Table 2 Material properties of interface element

\begin{tabular}{lllr}
\hline Property & Symbol & Value & Unit \\
\hline Normal stiffness & $k_{n}$ & $44.0 \times 10$ & $\mathrm{GPa}$ \\
Shear stiffness & $k_{s}$ & 22.0 & $\mathrm{GPa}$ \\
Cohesion & $\mathrm{C}$ & 10.0 & $\mathrm{kPa}$ \\
Friction angle & $\phi$ & 35.0 & degree \\
\hline
\end{tabular}

Table 3 Material properties of wall element

\begin{tabular}{llll}
\multicolumn{4}{c}{ Table 3 } \\
\hline Property & Symbol & Value & Unit \\
\hline Shear Modulus & $G$ & $8.4 \times 10$ & $\mathrm{GPa}$ \\
Poisson's Ratio & $v$ & 0.3 & \\
Density & $\rho$ & 7.8 & $\mathrm{~g} / \mathrm{cm}^{3}$ \\
\hline
\end{tabular}

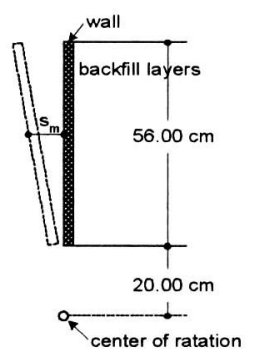

Fig. 6 Illustration of movement of the wall change of the lateral effective stresses, which is normal to the wall and the pore water pressure at the point $e-3$, is shown in Fig. 8. In this section the effective stress and the pore water pressure are expressed positive for compressive deformation. The effective stress is reduced due to the wall movement and the pore water increases at the beginning of the wall movement. These tendencies were observed in the experiment.

In Fig. 9 the dynamic pore water pressures acting on the wall at the point $e-1$ to $e-6$ are compared with the experimental values measured by the pore water cells. The dynamic pore water pressure corresponds

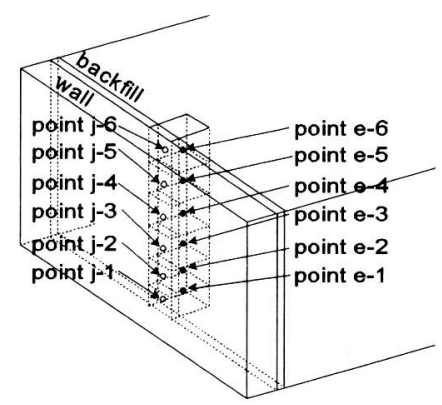

Fig.7 The positions of the picked up responses

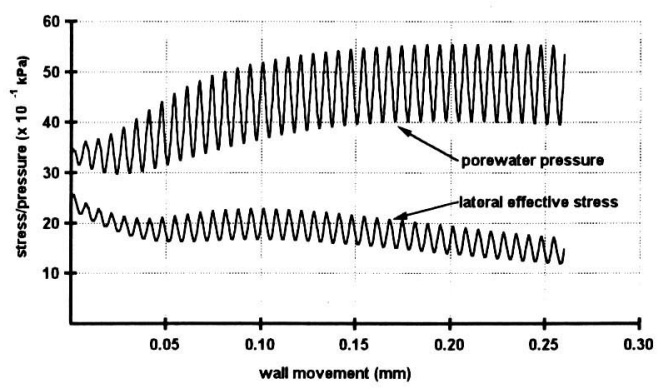

Fig. 8 Effect of the wall movement on the lateral effective stress and the pore water pressure

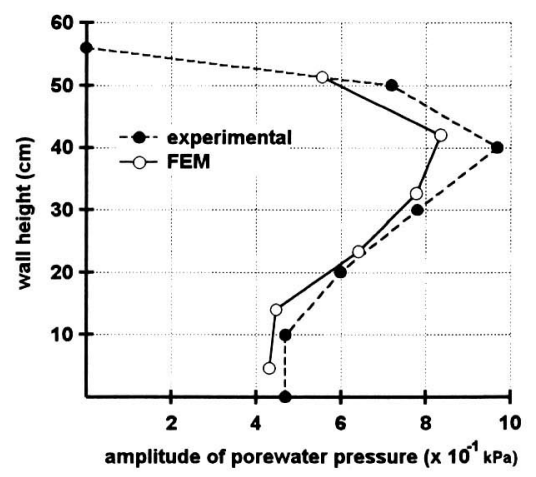

Fig.9 Comparison of the amplitude of the pore water pressure 
with the amplitude of the pore water pressures after ten seconds excitation when the wall movement is about $0.2 \mathrm{~mm}$. Fig. 9 shows the good agreement between the analysis and the experiment.

The pass of effective stress at point $e-1$ is plotted on the $I J$ plane in Fig. 10. The stress pass moves close to the critical state line according the increase of the wall movement. It is presumed the soil at the bottom of the soil layer is highly sheared and becomes close to the critical state when the wall moves outward. The shear stress-strain relation at point $e-1$, shown in Fig. 11, suggests the shear stress-strain relation has a nonlinear properties due to the wall movement. The effect of the dynamic loading is relatively small.

The numerical results of the effective lateral stress distribution at the wall are shown in Fig. 12 for the wall movement $0.2 \mathrm{~mm}$ in which the effective lateral stresses is the maximum in the one cyclic loading. In the experiments the lateral pressures were measured as the total force using the two load cells because it is not reliable to observe the small earth pressures by the earth pressure cells. The earth pressure distribution acting on the wall was not measured directly in the experiments. The experimental values are plotted in Fig. 12 assuming the hydrostatic distribution. The total areas of the numerical results and the experimental ones are close. It is suggested the total force predicted by the analysis coincides well with the experimental one.

In the experiments the value of the lateral earth

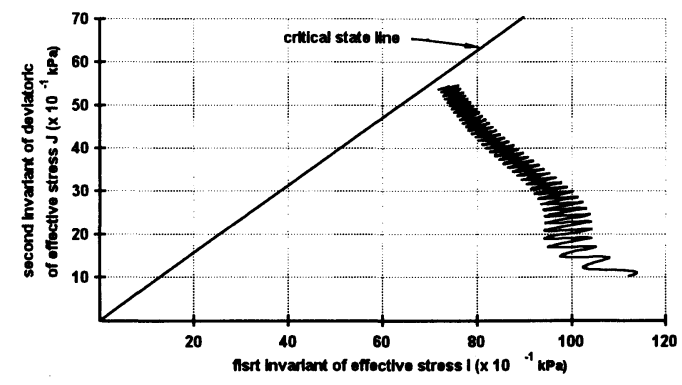

Fig.10 Effective stress path at Point $e-1$

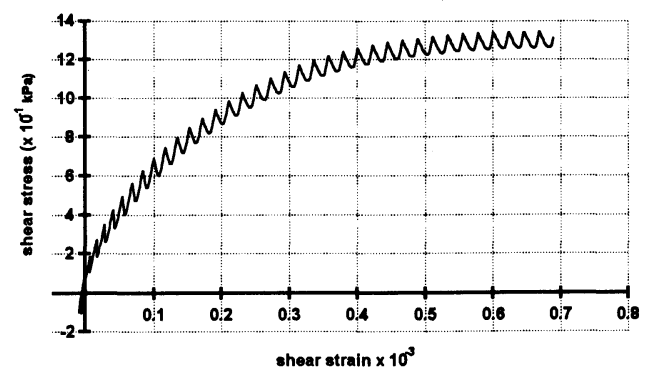

Fig.11 The Response of the shear stress-strain at Point $e-1$ pressures was defined as the seismic active earth pressure based on the study on the active earth pressures $^{12)}$. The slope of the experimental values in Fig. 13 represents the active earth pressure coefficient during earthquake, $K$, which is applied for the current design criterion of the quay walls. The value of $K$ in the experiment was 0.45 and nearly equals to the value calculated by the Mononobe-Okabe equation using the seismic coefficient $K$ and the density of the soil considering the effect of buoyancy. The value obtained by the analysis is 0.43 .

For the experimental data, the relative height of the applied point of the total force acting on the wall $h / H$ is calculated solving the equilibrium equation of the moment for rotation center of the wall.

The effect of the wall movement for $K$ and $h / H$ are shown in Fig. 13. According to the wall movement $K$ is reduced and $h / H$ is also reduced at first and turned to increase after the wall movement $s_{m}=0.06 \mathrm{~mm}$. The coefficient of the wall friction $f_{w}$ in the analysis is increased due to the wall movement, as shown in Fig.14. These characteristics for $K, h / H$ and $f_{w}$ are also confirmed in the experiments.

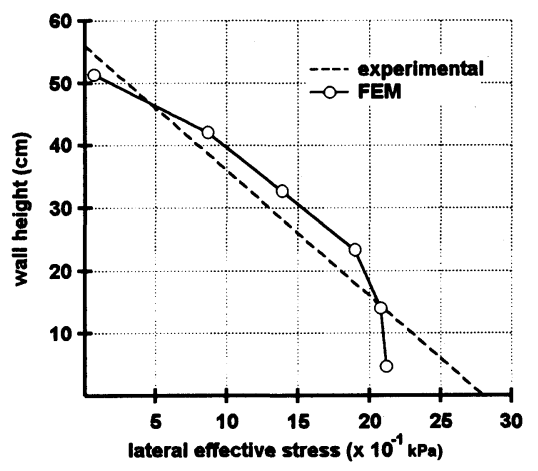

Fig.12 Comparison of the lateral pressure distribution

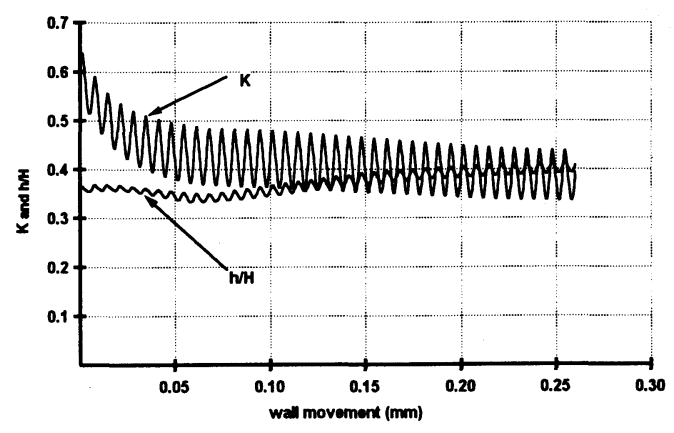

Fig.13 Responses of the earth pressure parameters 


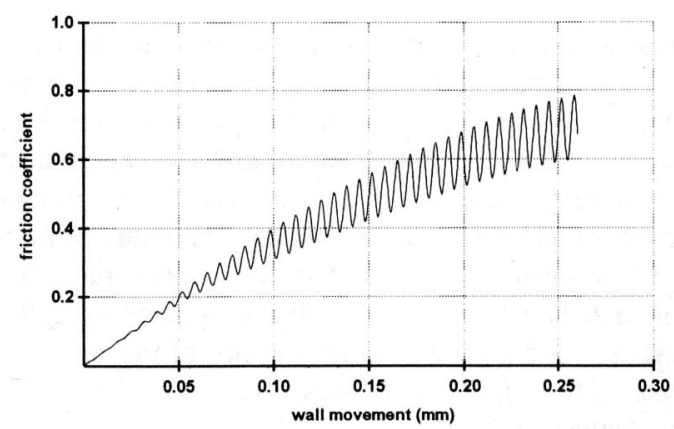

Fig.14 The response of the friction coefficient

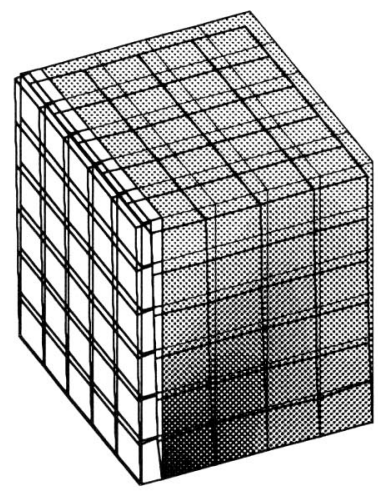

$2^{0}$

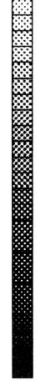

Fig.15 Deformation and failure mode of subdomain 1

The distribution of $\rho_{\phi}$ which denotes the tangent of the mobilized angle of friction similar with $\varsigma_{c r}$ is shown in Fig. 15, for the subdomain $l$ in the saturated soil layer and the wall movement $s_{m}=0.2 \mathrm{~mm}$. The darker points represent the stress conditions close to the critical state. The darker areas starting from the bottom is presumed as a failure zone in the backfill soil. It is difficult to define the failure line in the saturated soils. In the experiment the active state where the failure occurs was defined for the wall movement $s_{m}=0.2 \mathrm{~mm}$ but it is not confirmed by this numerical analysis because of the accuracy of the analysis close to the failure surface and the limitation of the infinitesimal small strain analysis.

\section{b) Performance of parallel computation}

To evaluate the performance of the nonlinear parallel computation, series of the analysis were carried out and results are shown in Tables 4 and 5 . In the series of the analysis the results for nonlinear soil materials are compared with those of linear soil material. In both of the linear and the nonlinear cases the model size and the number of processors are increased keeping the specified number of elements assigned to one processor constant. The subdomain for each processor is composed of 392 elements and 3072
Table 4 Performance of parallel computation

\begin{tabular}{lllll}
\hline $\begin{array}{l}\text { Num of } \\
\text { Procs. }\end{array}$ & Prob & Memory (GB) & Executing & Time $(\mathrm{sec})$ \\
\cline { 4 - 5 } & & & Linear & Nonlinear \\
\hline 1 & $1 \times 392$ & 5.406 & 71.066 & 204.148 \\
2 & $2 \times 392$ & 5.410 & 75.782 & 206.019 \\
4 & $4 \times 392$ & 5.418 & 80.933 & 212.539 \\
8 & $8 \times 392$ & 5.434 & 82.497 & 225.473 \\
16 & $16 \times 392$ & 5.468 & 84.007 & 230.981 \\
\hline
\end{tabular}

Table 5 Parallel computation indicators

\begin{tabular}{lllll}
\hline \multirow{2}{*}{$\begin{array}{l}\text { Scale of } \\
\text { Model }\end{array}$} & $\begin{array}{l}\text { Increment of } \\
\text { Memory (\%) }\end{array}$ & \multicolumn{2}{l}{ Increment of } & Executing Time(\%) \\
\cline { 3 - 4 } & & Linear & Nonlinear \\
\hline 1 & 0.000 & 0.000 & 0.000 \\
2 & 0.068 & 6.635 & 0.916 \\
4 & 0.222 & 13.884 & 4.110 \\
8 & 0.506 & 16.084 & 10.446 \\
16 & 1.143 & 18.210 & 13.144 \\
\hline
\end{tabular}

numbers of degrees of freedom. The executing time and the computer memory requirement were investigated as the parallel computation indicator. These indicator values were recorded after the first step of the dynamic analysis. The execution time is sum of the elapsed time for the calculation processes in each processor and the inter-processor communications. The memory size in Table 4 represents the maximum value for all the processors .

The percentage of the increment of the computer memory requirement and the executing time for each scale of the model are shown in Table 5. For the model memory requirement are $13.1 \%$ and $1.1 \%$,respectively in the nonlinear case. The increment of the executing time in the linear case is $18.2 \%$. These results suggests that the performance of the parallel computation is very efficient and more effective for nonlinear analysis than the linear case.

\section{CONCLUSIONS}

The followings are obtained as the results of this study.

(1). The 3D nonlinear FEM formulation for the dynamic response of saturated layers was derived simplifying the bounding surface model developed by Wolf and Crouch. This formulation is applicable to dynamic soil-structure interaction problems for saturated soil layers and time history response analysis.

(2). The 3D nonlinear FEM formulation was solved by the parallel processing procedure to reduce the computation time and the memories for 3D and nonlinear FEM models. In the implementation Domain Decomposition Method was applied and the Conjugate Gradient algorithm was used to solve the displacement in the whole domains introducing the modified Newton-Raphson proce- 
dures.

(3). The parallel processing FEM was applied to simulate the dynamic earth pressure of a saturated soil which was observed in the shaking table tests and affected by the lateral movement during the excitations. The dynamic pore water pressure acting on the wall by this analysis coincides well with those of the experimental data as shown in Fig. 9. The numerical value for the total force of seismic active earth pressures acting on the wall, which is the effective stress, was close to the experimental values as shown in Fig. 12. The validity of the numerical results was confirmed by the comparison with the experimental data.

(4). The efficiency of the parallel processing algorithm was made clear by the comparison of the executing time for different sizes of the model.

(5). The 3D nonlinear parallel FEM analysis was applied successfully to the seismic active earth pressure problem first by this study. It is also first analytical study to show the relation of the effective stresses and pore water pressures in the saturated soil layer under cyclic loading and with the effect of the wall movement.

\section{REFERENCES}

1) Dafalias, Y.F.: Bounding Surface Plasticity I, Mathematical Foundation and Hypoplasticity, J. Engg. Mech. Geomech, Vol. 112, No. 12, pp. 966-987, 1986.

2) Crouch, R. S. and Wolf, J.P.: Unified 3D Critical State Bounding Surface Plasticity Model for Soils incorporating Continues Plasticity Loading Under Cyclic Path, Constitutive Relations, Int. J. Num. Anal. Met., Geomech. Vol. 18, pp. 735-758, 1994.

3) Kawamura, M. and Tanjung, J.: Parallel FEM Analysis for Dynamic Responses of Saturated Soil Layer, J. Struc. Engg. JSCE, Vol .46A, pp. 703-712, 2000.

4) Papadrakakis, M.: Domain Decomposition Techniques for Computational Structural Mechanic, Parallel Solution Method in Computational Method (Ed. Papadrakakis, M), John Wiley \& Sons, Chichester, 1997.

5) Tallex, P.L. and Vidrascu, M.: Solving Large Scale Structural Problems on Parallel Computers Using Domain Decomposition Techniques, Parallel Solution Method in Computational Method (Ed. Papadrakakis, M), John Wiley \& Sons, Chichester, 1997.
6) Press, W.H., Tuekolsky, S.A., Vetterling, W.T. and Flannery, B.P.: Numerical Recipes in FORTRAN, Cambridge University Press, Melbourne, 1995.

7) Ghaboussi, A.M. and Wilson, E.L.: Seismic Analysis of Earth Dam Reservoir System, J. Soil Mech. And Found. Div., ASCE., Vol.99, No. SM10, pp. 849-862, 1973.

8) Zienkiewicz, O.C and Shiomi, T.: Dynamic Behavior of Saturated Porous Material, The Generalized Biot Formulation and Its Numerical Solution, Int. J. Num. Anal. Meth. Geomech., Vol. 8, pp. 71-96, 1984.

9) Zienkiewicz, O.C and Taylor, R.L.: The Finite Element Method, McGraw-Hill, London 1991.

10) Beskos, D. E. and Anagnostopoulos, S.A. (Editors): Computer Analysis and Design of Earthquake Resistant Structures: a Handbook, Computational Mechanics, Southampton, 1997.

11) Geist, A., Beguelin, A., Dogarra, J., Jiang, W., Macheck, R. and Sunderam, V.: PVM, Parallel Virtual Machine, A User's Guide and Tutorial for Networked Parallel Computing, The MTT Press, Massachusetts, 1996.

12) Kawamura, M.: Studies on Lateral Earth Pressures on Retaining Walls during Earthquakes and Heavy Rainfalls (in Japanese), Dissertation of Dr. Eng., Nagoya University, Nagoya, 1979.

13) Beer, G.: An Isoparametric Joint/Interface Element for Finite Element Analysis, Int. J. Num. Meth. Engg., Vol. 21, pp.585-600, 1985.

14) Toki, K., Sato, T. and Miura, F.: Separation and Sliding Between Soil and Structure During Strong Ground Motion, Earthquake Engg. Struc. Dyn., Vol. 9, pp. 263-277, 1981.

15) Been, K., Jefferies, M.G. and Hachey, J.: The Critical State of Sands, Geotechnique, Vol. 41, No. 3, pp. 365-381, 1991

16) Pradhan, B.S., Tatsuoka, F. and Sato, Y.: Experimental Stress-Dilatancy Relations of Sand Subjected to Cyclic Loading, Soil and Foundations, JSSMFE, Vol. 29. No.1 pp. 45-64, 1989.

17) Ishihara, K.: Soil Behavior in Earthquake Geotechnics, Clarendon Press, Oxford, 1996.

18) Crouch, R. S. and Wolf, J.P.: Unified 3D Critical State Bounding Surface Plasticity Model for Soils incorporating Continues Plasticity Loading Under Cyclic Path, Calibration and Simulation, Int. J. Num. Anal. Met., Geomech. Vol. 18, pp. 759-784, 1994.

19) Hazarika, H. and Matsuzawa, H.: Coupled Shear Band Method and Its Application to Seismic Earth Pressure Problem, Soils and Foundation, JSSMFE, Vol. 37, No. 3, pp. 65-77, 1997.

(Received May 7,2001) 
飽和土層の地震時土圧に関する 3 次元非線形並列有限要素解析

\section{河邑眞・Jafril Tanjung}

この論文では, 飽和土層における動的相互作用問題に対する, 3 次元非線形並列有限要素 解析方法を示す. 飽和土の繰り返し載荷時におりる非線形な力学的挙動を表現するため に, Bounding Surface Mode1 を用いた. また， 3 次元非線形動的解析の過大な計算負荷を軽 減するために，有限要素並列演算アルゴリズムには， Domain Decomposition Method と Conjugation Gradient Method を用いた. つきに，提案された手法の妥当性を検証するため に, 振動台を用いた地震時土圧実験の数値シミュレーションを行った. 地震時主働土圧と間 隙動水圧に関する数値解析結果は実験結果と良い一致を示した. 